\title{
Temperature effect in the conductance of hydrogen molecule
}

\author{
M. Crisan and I. Grosu \\ Department of Theoretical Physics, University of Cluj, 3400 Cluj-Napoca, Romania
}

\begin{abstract}
We present a many-body calculation for the conductance of a conducting bridge of a simple hydrogen molecule between $P t$ electrodes. The experimental results showed that the conductance $G=d I / d V$ has the maximum value near the quantum unit $G_{0}=2 e^{2} / h$. The $I-V$ dependence presents peak and dip and we consider that the electron-phonon interaction is responsible for this behavior. At $T=0$ there is a step in this dependence for the energy of phonons $\omega_{0}$ which satisfies $e V=\omega_{0}$. We calculated the conductance at finite temperature and showed that $d G(T) / d V \propto$ $1 / 4 T \cosh ^{2} \frac{e V-\omega_{0}}{2 T}$.
\end{abstract}

\section{INTRODUCTION}

The recent advances in manipulation of single molecules permit to measure the transport properties of a setup formed from an individual molecule between two electrodes. In contrast to the quantum dots, based on the semiconducting islands, the molecular devices have a more complicated electronic structure.

In a remarkable experiment [1] a setup consisting from a single hydrogen molecule between $\mathrm{Pt}$ electrodes was measured and the conductance behavior demonstrated the influence of the vibrational degree of freedom in the transport. The effect was also observed in various organic molecules [2], carbon nanotubes [3] fulerenes [4] but a theoretical description of these systems is more complicated because of the energy spectrum of the electrons from these molecules. The simplest model which is realistic for the hydrogen molecule setup is to consider the coupling between the vibrational mode (considered as phononic) of frequency $\omega_{0}$ and electrons from the leads. The main effect which appears in the I-V characteristic is the occurrence of a step at $V=\omega_{0} / e$, which corresponds to dip versus peak in $d^{2} I / d V^{2}$. Such a behavior has been studied also in [5, 6, 7]. Many models have been proposed by different authors [8, 9, 11] in order to explain the influence of vibrational modes on the transport, but only recently Egger and Gogolin [12] presented an analytical calculation explaining the current-voltage relation. Their calculation, which gives the correction to the current $\delta I$,is a perturbative calculation for the electron-phonon interaction and takes only the $g^{2}$ contributions similar to the approximation from Ref. 13, 14] where the electron-phonon interaction is treated in the weak coupling approximation. In a higher order of perturbation theory $g^{n}$ the step behavior is expected at $n \omega_{0}$ - The occurrence of the step feature at multiples of $\omega_{0}$ is related to the strong- coupling picture using the polaron transformation [15].

In this paper we present a many- body calculation of the finite temperature conductance for the hydrogen molecule between metallic leads. The procedure is similar to this from Ref. [12] but we calculate the conductance of the setup close to the characteristic frequency $\omega_{0}$ at finite temperature. The peak (dip)which appear at this frequency has a finite width, due to thermal effect.

The paper is structured as follows. In Sec.II we present the model and calculate the Green functions. In Sect.III we calculate the lowest order correction $\delta I$ to the current which is given by the electron -phonon processes. The concluding remarks are presented in Sec.IV.

\section{MODEL AND GREEN FUNCTION}

We start with the Hamiltonian which describes the interaction between electrons and the Holstein phonos used in Ref. 7] for the molecular dots and in Ref. 13] for study of the inelastic scattering influence on the the tunnelling current in the two dimensional systems. The simple Hamiltonian, which is analytically tractable, has the form :

$$
H=H_{0}+H_{i}
$$

where

$$
\begin{gathered}
H_{0}=\epsilon_{0} d^{\dagger} d+\omega_{0} b^{\dagger} b+\sum_{k \alpha}\left(\epsilon_{k}-\mu_{\alpha}\right) c_{k \alpha}^{\dagger} c_{k \alpha} \\
H_{i}=\sum_{k, \alpha}\left(V_{\alpha} d^{\dagger} c_{k \alpha}+H . c\right)+g Q d^{\dagger} d
\end{gathered}
$$

where $Q=b+b^{\dagger}$.

In the Hamiltonian (1) $d$ and $d^{\dagger}$ are the operators for the single level $\epsilon_{0}$, the Holstein phonons with energy $\omega_{0}$ are described by the operators $b^{\dagger}$ and $b$, the electrons with the energy $\epsilon_{k \alpha},(\alpha=L, R)$ and chemical potential $\mu_{\alpha}$ are described by the operators $c_{k \alpha}$ and $c_{k \alpha}^{\dagger}$. The interaction between the electrons from the leads and the impurity is $V_{\alpha}$ and $g$ describes the interaction between the localized electronic level $\epsilon_{0}$ and the phonons. This Hamiltonian has been used by Egger and Gogolin [12] in this problem at $T=0$.Using the equation of motion method we calculate the Green function $G_{0}^{r}=<<d \mid d^{\dagger}>>$ as $G_{0}^{r}(\omega)=\left(\omega-\epsilon_{0}+i \Gamma\right)^{-1}$ where we define $\Gamma=\Gamma_{L}+\Gamma_{R}$ and $\Gamma_{\alpha}=\pi N(0)\left|V_{\alpha}\right|^{2}$. The Green function describing the system is :

$$
G^{r}(\omega)=G_{0}^{r}(\omega)+G^{r}(\omega) \Sigma^{r}(\omega) G_{0}^{r}(\omega)
$$

where the self energy $\Sigma^{r}(\omega)$ is taken in the lowest order and has the form[14]: 


$$
\Sigma^{r}(\omega)=-\frac{g^{2}}{2} \int d \omega^{\prime} d \omega^{\prime \prime} \frac{\left[\operatorname{Im} G_{0}\left(\omega^{\prime}, \epsilon_{0}\right) \operatorname{Im} D_{0}\left(\omega^{\prime \prime}, \omega_{0}\right)\right] A\left(T, \omega^{\prime}, \omega^{\prime \prime}\right)}{\omega^{\prime}+\omega^{\prime \prime}-\omega}
$$

where $A\left(T, \omega^{\prime}, \omega^{\prime \prime}\right)=1-f_{F}\left(\omega^{\prime}\right)+n_{B}\left(\omega^{\prime \prime}\right), f_{F}(\omega)$ is the Fermi function and $n_{B}(\omega)$ is the Bose function, and the phononic Green function $D_{0}\left(\omega, \omega_{0}\right)$ is :

$$
D_{0}^{R}\left(\omega, \omega_{0}\right)=\frac{1}{2 \omega_{0}}\left[\frac{1}{\omega-\omega_{0}+i \delta}+\frac{1}{\omega+\omega_{0}+i \delta}\right]
$$

From Eqs.(5-6)we obtain :

$$
\operatorname{Im} \Sigma^{r}(\omega)=-g^{2} \sum_{\alpha, s= \pm 1} \frac{\Gamma_{\alpha} f_{F}\left(\omega_{0}-s\left(\bar{\mu}_{\alpha}-\omega\right)\right)}{\left(\omega+s \omega_{0}\right)+\Gamma^{2}}
$$

where $\bar{\mu}=\left(\mu_{R}+\mu_{L}\right) / 2-\epsilon_{0}$ and $\bar{\mu}_{\alpha=L / R= \pm 1}=\bar{\mu} \pm \mathrm{eV} / 2$.

This contribution is dominant in the correction in current for small $\omega_{0} / \Gamma$ [12], and we will calculate only this contribution at finite temperature.

\section{CURRENT AND CONDUCTANCE}

The electrical current through the dot can be calculated from the Green function $G^{r}(\omega)$ as :

$$
I(V)=-\frac{4 e}{h} \frac{\Gamma_{L} \Gamma_{R}}{\Gamma} \int d \omega\left[f_{L}(\omega)-f_{R}(\omega)\right] \operatorname{Im} G^{r}(\omega)
$$

and for the case of $g=0$ we get the current $I_{0}$ as

$$
I_{0}(V)=\frac{e}{h} \frac{4 \Gamma_{L} \Gamma_{R}}{\Gamma}\left[\arctan \left(\bar{\mu}_{L} / \Gamma\right)-\arctan \left(\bar{\mu}_{R} / \Gamma\right)\right] .
$$

For $V \rightarrow 0$ the transparency of the junction, $\Upsilon=$ $\left(h / e^{2}\right) d I / d V$ is

$$
\Upsilon=\frac{4 \Gamma_{L} \Gamma_{R}}{\Gamma} \frac{1}{1+(\bar{\mu} / \Gamma)^{2}} \leq 1 .
$$

Using Eqs.(4-8) we calculate the correction to the current given by the inelastic electron-phonon scattering [12] as:

$$
\delta I_{\text {inel }}=\frac{e}{h} \frac{\Gamma_{L} \Gamma_{R}}{\Gamma} \int_{\bar{\mu}_{R}}^{\bar{\mu}_{l}} d \omega F(\omega, V)
$$

where $F(\omega)$ is given by :

$$
F(\omega, V)=-\frac{\Gamma^{2}-\omega^{2}}{\left(\omega+\Gamma^{2}\right)^{2}} \sum_{\alpha, s} g^{2} \Gamma_{\alpha} \frac{f_{B}\left[\omega_{0}-s\left(\bar{\mu}_{\alpha}-\omega\right)\right]}{\left(\omega+s \omega_{0}\right)+\Gamma^{2}} .
$$

In order to calculate the conductance $G=d I / d V$ and its derivative $d G / d I$ we will use the relation:

$\frac{d}{d V} \int_{\bar{\mu}_{R}}^{\bar{\mu}_{L}} F(\omega, V)=\frac{e}{2}\left[F\left(\bar{\mu}_{L}, V\right)+F\left(\bar{\mu}_{L}, V\right)\right]+\int_{\bar{\mu}_{R}}^{\bar{\mu}_{L}} d \omega \frac{d F}{d V}$.
Using this formula we calculate the derivative $d \delta I_{\text {inel }} / d V$ as :

$$
\frac{d \delta I_{\text {inel }}}{d V}=-\frac{e^{2}}{h} \frac{\Gamma_{L} \Gamma_{R}}{\Gamma} g^{2}\left[f_{F}\left(\omega_{0}-e V\right) S_{1}+\Theta\left(V-\frac{\omega_{0}}{e}\right) S_{2}\right]
$$

where :

$$
S_{1}=\sum_{\alpha= \pm 1} \frac{\Gamma_{\alpha}\left(\Gamma^{2}-\bar{\mu}_{-\alpha}^{2}\right)}{\left(\Gamma^{2}+\bar{\mu}_{-\alpha}^{2}\right)\left[\Gamma^{2}+\left(\bar{\mu}_{-\alpha}+\alpha \omega_{0}\right)^{2}\right]}
$$

and

$$
S_{2}=\sum_{\alpha= \pm 1} \frac{\Gamma^{2}-\left(\bar{\mu}_{\alpha}-\alpha \omega_{0}\right)^{2}}{\left(\bar{\mu}_{\alpha}^{2}+\Gamma^{2}\right)\left[\left(\bar{\mu}_{\alpha}^{2}-\alpha \omega_{0}\right)+\Gamma^{2}\right]^{2}} .
$$

Using these results we calculate the contribution of the inelastic scattering in $\frac{d G}{d V}$ as :

$$
\frac{d^{2} \delta I_{\text {inel }}}{d V^{2}}=-\frac{e^{2}}{h} \frac{\Gamma_{L} \Gamma_{R}}{\Gamma} g^{2} \frac{S_{1}+S_{2}}{4 T \cosh ^{2}\left(\frac{e V-\omega_{0}}{2 T}\right)} .
$$

This is the main result of this paper, which shows that at finite temperature the peak (dip) in the conductance derivative $d G / d V$ has a finite width at $e V=\omega_{0}$ at low , but finite temperature. At $T=0$ we reobtain the $\delta(e V-$ $\omega_{0}$ ) behavior predicted in Ref.[12].

The contribution of the elastic scattering present a logarithmic divergence at $e V=\omega_{0}$ which create symmetric dip or peak in the differential conductance. The relative importance of the inelastic versus quasi-elastic contributions has been analyzed in [12 at $T=0$ where was showed that at $\omega_{0} / \Gamma<<1$ and large $\bar{\mu}$ the inelastic channel is dominant. This result remain valid also at finite temperature and in the following we will discuss the conditions for the occurrence of a dip or peak in the inelastic correction. First we mention that this correction is not any more singular, as at $T=0$, but we obtain the result from [12] in this limit. For $\Gamma_{L}=\Gamma_{R}$ and $\bar{\mu}=0$ we have $\Upsilon=1$ for $\omega_{0}>2 \Gamma$ instead of a dip we get a peak. This is a particular case, and for $\bar{\mu} \neq 0$ and $\bar{\mu}>\sqrt{\Gamma^{2}+\omega_{0}^{2} / 4}$ we have a peak. We also have at $\bar{\mu}= \pm \Gamma$ the transparency $\Upsilon=1 / 2$ which is in fact the point of the peak-dip transition.

At $T=0$ the only way to obtain a finite life-time of phonons, which may give a smearing of the step and/or peak feature is to include the electronic polarization in the Green function of the phonons, as was suggested in 12. However, such a calculation implies the higher-order perturbations in $g$ and it is difficult to be performed analytically. In this paper the discussion concerning the occurrence of the step or/and peak is identically to that of the authors of [12], but the coupling to the thermal phonons generate in a natural way a smearing of the step or/and peak structure. 


\section{CONCLUDING REMARKS}

We analyzed, using the many body method, the transport in the simplest molecular dot consisting from a hydrogen molecule between the Pt leads. The analytical calculations of the effect given by the electron-phonon interaction on current at finite temperature have been performed.The obtained results can be regarded as complementary to the $T=0$ similar calculations presented in Ref.[12]. However, at zero temperature the concept of phonon is not defined and in the transport we use the concept of inelastic scattering. On the other hand the experimental results showed the existence of behavior in the $d G / d I$ which cannot be described by the simple $\delta\left(e V-\omega_{0}\right)$ behavior, which is specific for $T=0$. We showed that at finite temperature a dip or a peak in this quantity is described by $d G / d I=C\left(\bar{\mu}, \Gamma, \omega_{0}\right) 1 /(4 T$ $\left.\cosh ^{2} \frac{\mathrm{eV}-\omega_{0}}{2 T}\right)$. This behavior is given by the inelastic scattering between electrons and phonons, the elastic contribution giving a non relevant contribution to the transport in this system, which can be considered as a molecular quantum dot. Our results at $T=0$ are identically with the results from [12], and the signature the constant $C\left(\bar{\mu}, \Gamma, \omega_{0}\right)$ gives the same conditions. Our calculations completed the microscopic model presented in Ref. 12], but we consider that the transport in more complicated molecules is difficult to be treated analytically.However, the model can be a starting point for the study of transport in the more complex molecular systems.

We thank Alexander Gogolin for useful correspondence on the subject.
[1] R. H. M. Smit, Y. Noat, C. Untiedt, N.D. Lang, M.C. van Hemert, and J. M. van Ruitenbeek, Nature (London) 419, 906 (2002); D. Djukic, K. S. Thygesen, C. Untiedt, R. H. M. Smit, K.W. Jacobsen, and J. M. van Ruitenbeek, Phys. Rev. B 71, 161402(R) (2005).

[2] N. B. Zhitenev, H. Meng, and Z. Bao, Phys. Rev. Lett. 88, 226801 (2002); X. H. Qiu, G. V. Nazin, and W. Ho, Phys. Rev. Lett.92, 206102 (2004); L. H. Yu, Z. K. Keane, J.W. Ciszek, L. Cheng, M. P. Stewart, J. M. Tour, and D. Natelson, Phys. Rev. Lett 93, 266802 (2004).

[3] B. J. LeRoy, S. G. Lemay, J. Kong, and C. Dekker, Nature (London) 432, 371 (2004).

[4] A. N. Pasupathy, J. Park, C. Chang, A.V. Soldatov, S. Lebedkin,, R. C. Bialczak, J. E. Grose, L. A. K. Donev, J. P. Sethna, D. C. Ralph, and P. L. McEuen, Nano Lett. 5, 203 (2005).

[5] T. Mii, S.G. Tikhodeev, and H. Ueba, Phys. Rev. B 68, 205406 (2003).

[6] M. Galperin, M. A. Ratner, and A. Nitzan, J. Chem. Phys. 121, 11965 (2004).

[7] M. Galperin, M. A. Ratner, and A. Nitzan, J. Phys.: Condens. Matter 19, 103201 (2007)

[8] P. S. Cornaglia, H. Ness, and D. R. Grempel, Phys. Rev.
Lett. 93, 147201 (2004); P.S. Cornaglia, D.R. Grempel, and H. Ness, Phys. Rev. B 71, 075320 (2005); L. Arrachea and M.J. Rozenberg, Phys.Rev. B 72, 041301(R) (2005); C. . Balseiro, P.S. Cornaglia, and D. R. Grempel, Phys. Rev.B 74, 235409 (2006).

[9] L. de la Vega, A. Martin-Rodero, N. Agrait, and A. Levy Yeyati, Phys. Rev. B 73, 075428 (2006).

[10] A. Donarini, M. Grifoni, and K. Richter, Phys. Rev. Lett. 97, 166801 (2006).

[11] A. Zazunov, D. Feinberg, and T. Martin, Phys. Rev. Lett. 97, 196801 (2006); Phys. Rev. B 73, 115405 (2006); A. Zazunov, R. Egger, C. Mora, and T. Martin, Phys. Rev. B 73, 214501 (2006); A. Zazunov and T. Martin, Phys. Rev. B 76, 033417 (2007).

[12] R. Egger and A. O. Gogolin, Phys. Rev. B 77, 113405 (2008).

[13] Fransson an A. Balatsky Phys. Rev. B, 75, 195337 (2007).

[14] S. Engelsberg and J.R. Schrieffer, Phys. Rev. 131, 993 (1963).

[15] A. S. Alexandrov and A. M. Bratkovsky, Phys. Rev. B 67, 235312 